\title{
Self-Timed Scheduling Analysis for Real-Time Applications
}

\author{
Orlando M. Moreira and Marco J. G. Bekooij \\ NXP Semiconductors Research, 5656 AE Eindhoven, The Netherlands
}

Received 1 September 2006; Accepted 2 April 2007

Recommended by Roger Woods

\begin{abstract}
This paper deals with the scheduling analysis of hard real-time streaming applications. These applications are mapped onto a heterogeneous multiprocessor system-on-chip (MPSoC), where we must jointly meet the timing requirements of several jobs. Each job is independently activated and processes streams at its own rate. The dynamic starting and stopping of jobs necessitates the usage of self-timed schedules (STSs). By modeling job implementations using multirate data flow (MRDF) graph semantics, real-time analysis can be performed. Traditionally, temporal analysis of STSs for MRDF graphs only aims at evaluating the average throughput. It does not cope well with latency, and it does not take into account the temporal behavior during the initial transient phase. In this paper, we establish an important property of STSs: the initiation times of actors in an STS are bounded by the initiation times of the same actors in any static periodic schedule of the same job; based on this property, we show how to guarantee strictly periodic behavior of a task within a self-timed implementation; then, we provide useful bounds on maximum latency for jobs with periodic, sporadic, and bursty sources, as well as a technique to check latency requirements. We present two case studies that exemplify the application of these techniques: a simplified channel equalizer and a wireless LAN receiver.
\end{abstract}

Copyright (c) 2007 O. M. Moreira and M. J. G. Bekooij. This is an open access article distributed under the Creative Commons Attribution License, which permits unrestricted use, distribution, and reproduction in any medium, provided the original work is properly cited.

\section{INTRODUCTION}

\subsection{Application domain}

In order to deliver high-quality output, streaming media applications have tight real-time (RT) requirements. These typically come in several different flavors [1], according to the type of requirements. For instances, in hard real time the deadlines of jobs cannot be missed, while in soft real time the deadlines can be missed, but the rate of misses must be kept below a specified maximum. Because human perception is more tolerant of frame loss in a video signal than sample loss in an audio signal, video applications are often implemented as soft real time, while most radio and audio applications are treated as hard real time.

Contrarily to real-time control applications where most temporal requirements are in terms of latency, the temporal requirements of real-time streaming applications are mostly throughput oriented, although latency requirements may still be present.

Embedded platforms for streaming are expected to handle several streams at the same time, each with its own rate. Typically, functionality can be divided in minimal groups of interconnected tasks that can be started and stopped inde- pendently by an external source such as the user. We refer to such groups of tasks as jobs. The connections among tasks within a job are static. Jobs can be connected dynamically to each other by feeding the output of one as an input to another. This is done, for instance, when equalization is applied to the output of an audio decoder. The number of use cases (a use case is a combination of simultaneously executing job instances that the device must support) is potentially very high.

This application domain includes car infotainment [2], where the user can request at any moment radio baseband processing for either AM or FM, or digital decoding or encoding for one of many audio formats. Several streams can be present at a time, both because independent sound output must be provided to front and backseat, and different streams may be mixed (such as when listening to music while receiving a phone call). Moreover, further sound processing may be provided, such as equalization or echo cancellation for a hands-free phone kit.

\subsection{Hardware issues}

For embedded hardware platforms, multiprocessor systemson-chip (MPSoCs) provide a good balance between cost, 
power efficiency, and flexibility. These systems are typically heterogeneous, as the usage of application-specific coprocessors can greatly improve performance at low area cost. Also, it is likely that the need for scalability and ease of design will drive these systems towards the usage of uniform networkson-chip (NoCs) [3] for interprocessor communication.

In order to allow maximum flexibility at the lowest cost, jobs share computation, storage, and communication resources. This poses a particularly difficult problem for the programming of real-time applications. Resource sharing leads to uncertainty of resource provision which can make the system noncomposable, that is, the temporal behavior of each job becomes dependent on other jobs and cannot be verified in isolation. As the current software verification processes rely strongly on extensive simulation, a large number of tests would have to be carried out in order to verify all possible use cases. Even if this were feasible, few guarantees could be given, since the simulation results only apply for the particular data streams that were used for testing. This is unacceptable for hard-real-time systems.

We are convinced that the solution to these mounting problems requires a shift to a more analytical, model-based approach. Although we do believe that extensive testing and simulation may still be necessary in many cases, we try to see how far we can go with a strictly model-based approach. This is, however, far more than a theoretical exercise. In fact, the techniques we developed are being applied to the design of an MPSoC for a next-generation Car Infotainment system [2].

\subsection{Our approach}

\subsubsection{Hardware requirements}

Our model-based approach can only be exploited to the fullest when some restrictions are imposed to the hardware. We think that these restrictions become necessary to make the design of complex MPSoCs manageable.

Under this perspective, the most desirable feature of an MPSoC is resource virtualization: a job may see and use only the part of the system resources that is reserved for it. This implies that resource reservation is done a priori. Virtualization makes a system composable. Because of this, we prescribe the usage of networks-on-chip (NoCs) such as the Æthereal $[3,4]$, which allow the definition of connections with guaranteed throughput and latency, therefore isolating each communication channel from the rest of the system. To enable virtualization, all arbitration mechanisms in the SoC should provide guarantees of a rather tight upper bound to the waiting time for resource provision. We refer to such arbitration mechanisms as predictable.

In this paper, we restrict ourselves to SoCs where every processor has its own local dedicated memory. Shared memory can be handled by our analysis techniques, but its access must be arbitrated in a predictable fashion. We assume that caches are not used. This is fine for hard-real-time applications, since little advantage can be taken from the probabilistic performance improvement offered by caching. Again, the next-generation Car Infotainment system we use as case

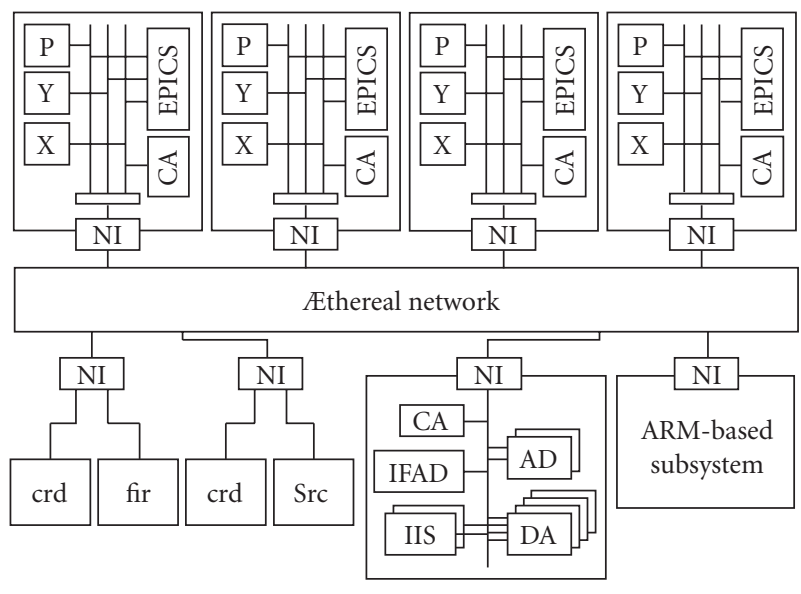

FIGURE 1: Next-generation car infotainment system architecture.

study serves as practical example of a SoC that fully meets our requirements for effective analysis. The architecture of this system is depicted in Figure 1.

Each EPIC DSP core in Figure 1 has its own data $(X, Y)$ and program $(\mathrm{P})$ memories and a communication assist (CA) unit that moves data to and from the network such that intertile communication is decoupled from computation; there are FIR and Cordic accelerators, a peripherals tile with input and output devices such as D/A and A/D converters, and an ARM for general control, resource management, and user interaction. All subsystems are connected via network interfaces (NIs) to an Æthereal NoC. Each NI has a number of input and output queues with limited buffer capacity. Tiles communicate by establishing one-way connections through the network. Tasks post data in a buffer in the local memory. These data are pushed into an NI queue by the CA and transported by the network to the receiving NI, where the local CA transfers the data to a buffer in the local memory. A credit mechanism guarantees that no information is driven into the network without buffer space being available at the receiving NI. As the Æthereal network provides guaranteed throughput connections [3], this communication channel is immune to interference from other communication.

\subsubsection{Run-time scheduling}

For systems that execute multiple hard-RT and soft-RT jobs that process independent streams, fully static or static order scheduling are not sufficient. This is for two reasons. First, the fact that jobs both start and stop independently would require a schedule computed at design time for every combination of jobs that can be active simultaneously. Second, it may be that there are soft-RT jobs running in the system, and these can require a number of executions that is dependent on the value of the input data. As a consequence, a schedule cannot be computed at design time and processor sharing requires run-time task scheduling. 
Fully dynamic scheduling also has drawbacks. It adds overhead to the system and requires a centralized task dispatch queue (a global, nonscalable resource). Moreover, its benefits can only be exploited if task migration can be done efficiently, which is complex under distributed memory architecture (which also means it does not scale up well). Therefore, we allow task-to-processor assignment to be done either at compile or at run time, but task migration is not supported. At job start, tasks are assigned on the fly to processors by a resource manager, similar to the ones we propose in $[5,6]$. The task scheduling on a processor at run time must be predictable. Both preemptive and non-preemptive scheduling mechanisms are considered, because it is common for weakly programmable application-specific processors not to support preemption.

\subsubsection{Job mapping}

Thanks to resource virtualization, we can map jobs independently to the MPSoC. In our constraint-driven model-based approach, there are two types of constraints: the throughput and latency constraints of the jobs and the hardware constraints imposed by the architecture of the MPSoC. The inputs to job mapping are a functional specification, a set of temporal requirements, and a description of the MPSoC instance. The output is an implementation of the functionality on the MPSoC that is guaranteed to meet the temporal requirements. Task assignment and static ordering may be specified, or, in alternative, a relative deadline per task to be guaranteed by the local scheduler of a processor.

We use an iterative mapping process where implementation decisions taken in one iteration become part of the set of constraints for the next. We do not enforce a single flow because the steps needed to come from functional specification to output do not follow a unique, predefined order. This is to account for the fact that each application poses different challenges and a one-size-fits-all approach may be counterproductive to the objective of finding the most cost-effective solutions. We also assume that although the design is assisted by tools, and almost all steps can be made fully automatic, manual intervention of the designer may sometimes be required.

\subsection{Analysis model}

As mentioned in the previous section, our constraint-driven methodology is model based, that is, constraint checking is enabled by the ability to generate a joint model of computation, communication, and resource sharing, which in turn allows us to verify temporal constraints.

We use multirate data flow (MRDF) [7] as our model semantics. It fits the application domain well because, while use cases are dynamic, jobs typically have data-driven static structures that can be expressed in MRDF. As we will show in this paper, MRDF provides the necessary analytical properties that allow temporal analysis of a complete or partial mapping at design time. In Section 4 we show how we are able to use MRDF to model jointly computation, communication, and arbitration mechanisms.

We do not limit ourselves to jobs expressed in MRDF. There is functionality that cannot be expressed in a straightforward way in MRDF that we can still model and analyze. In such a case, however, model construction requires insight into both the application and the semantics of MRDF.

Traditionally, temporal analysis of self-timed schedules of MRDF graphs only aims at evaluating the average throughput [8]. It cannot cope with latency constraints or constraints that result from the interfacing of the system with its environment. In this paper we present new techniques that partially remove these limitations, by elaborating on the monotonic property of MRDF and the relation between self-timed and static periodic schedules.

\subsection{Related work}

Our analysis model resembles the model presented in [9]. In that paper, edges can be used to represent sequence constraints between computation actors allocated to the same processor, while additional actors can be used to account for communication times, while MRDF analysis is used to check whether the self-timed implementation meets the throughput constraint. However, run-time scheduling is not modeled. Even more importantly, no analysis or enforcement means are provided for latency or strictly periodic execution requirements of sources and sinks of jobs.

In [8], latency is defined as the time elapsed between periodic source and sink execution. This book also shows how this can be calculated by symbolic simulation of the worstcase self-timed schedule of the job graph. Such an approach is not without problems. One problem is that it requires symbolic simulation of the job graph, which is in general untrackable, even for single-rate data flow graphs. Moreover, this definition is not as general as ours, since it only works if there is at least one path between source and sink without delays, and it only works if sources are periodic.

In [10], latency and buffer sizing are studied in the context of PGM graphs, which are comparable in expressivity to MRDF graphs. The analysis done in this work, however, limits itself to graphs with chain topology. Moreover, [10] does not allow for feedback loops, does not model interprocessor communication, requires EDF scheduling and a strictly periodic source.

The event model used in the SYMTA/S tool [11] cannot cope with critical cycles (i.e., they are not taken into account). Latency is only measured as a result of mapping, never taken into account as a constraint during the mapping processes; the same holds for buffer sizes.

\subsection{Paper organization}

In Section 2, we present our notation and some important properties of the MRDF model. We also state why we can restrict ourselves to the analysis of single-rate data flow (SRDF) graphs without loss of generality. In Section 3, we elaborate on the relation between self-timed and periodic execution 
of SRDF graphs. Expressing hardware resource constraints in the MRDF model is discussed in Section 4. Our main contribution is presented in Section 5 in which we address the issue of interfacing a job with its environment. The case studies in Section 6 illustrate the use of our analysis techniques. In the last section, we state the conclusions.

\section{MRDF NOTATION AND PROPERTIES}

In this section, we present our notation, some properties of MRDF graphs, and the relation between MRDF graphs and SRDF graphs. This is a reference material and can, for the most, be found elsewhere in the literature $[7,8,12]$.

\subsection{Graphs}

A directed graph $G$ is an ordered pair $G=(V, E)$, where $V$ is the set of vertices or nodes and $E$ is the set of edges or arcs. Each edge is an ordered pair $(i, j)$ where $i, j \in V$. If $e=(i, j) \in E$, we say that $e$ is directed from $i$ to $j . i$ is said to be the source node of $e$ and $j$ the sink node of $e$. We also denote the source and sink nodes of $e$ as $\operatorname{scr}(e)$ and $\operatorname{snk}(e)$, respectively.

\subsection{Paths and cycles in a graph}

A path in a directed graph is a finite, nonempty sequence of edges $\left(e_{1}, e_{2}, \ldots, e_{n}\right)$ such that $\operatorname{snk}\left(e_{i}\right)=\operatorname{scr}\left(e_{i+1}\right)$, for $i=1,2, \ldots, n-1$. We say that path $\left(e_{1}, e_{2}, \ldots, e_{n}\right)$ is directed from $\operatorname{scr}\left(e_{1}\right)$ to $\operatorname{snk}\left(e_{n}\right)$; we also say that this path traverses $\operatorname{scr}\left(e_{1}\right), \operatorname{scr}\left(e_{2}\right), \ldots, \operatorname{scr}\left(e_{n}\right)$; the path is simple if each node is only traversed once, that is $\operatorname{scr}\left(e_{1}\right), \operatorname{scr}\left(e_{2}\right), \ldots, \operatorname{scr}\left(e_{n}\right)$ are all distinct; the path is a circuit if it contains edges $e_{k}$ and $e_{k+m}$ such that $\operatorname{scr}\left(e_{k}\right)=\operatorname{snk}\left(e_{k+m}\right), m \geq 0$; a path is a cycle if it is simple and $\operatorname{scr}\left(e_{1}\right)=\operatorname{snk}\left(e_{n}\right)$.

\subsection{Multirate data flow graphs}

A multirate data flow (MRDF) graph-also known as synchronous data flow $[7,8]$ - is a directed graph, where nodes are referred to as actors, and represent time consuming entities, and edges (called arcs) represent FIFO queues that direct values from the output of an actor to the input of another. Data is transported in discrete chunks, referred to as tokens. When an actor is activated by data availability it is said to be fired. The condition that must be satisfied such that the actor may be fired is called the firing rule. MRDF prescribes strict firing rules: the number of tokens produced (consumed) by an actor on each output (input) edge per firing is fixed and known at compile time. During an execution of a data flow graph, all the actors may get fired a potentially infinite amount of times. Actors have a valuation $t: V \rightarrow \mathbb{N} ; t(i)$ is the execution time of $i$. Arcs have a valuation $d: E \rightarrow \mathbb{N} ; d(i, j)$ is called the delay of $\operatorname{arc}(i, j)$ and represents the number of initial tokens in $(i, j)$.

Arcs have two more valuations associated with them: prod $: E \rightarrow \mathbb{N}$ and cons $: E \rightarrow \mathbb{N}$. $\operatorname{prod}(e)$ gives the constant number of tokens produced by $\operatorname{scr}(e)$ on $e$ in each firing and cons $(e)$ gives the constant number of tokens consumed by $\operatorname{snk}(e)$ in each firing. An MRDF can be completely defined by a tuple $(V, E, t, d$, prod, cons). We are interested in applications that process data streams, which typically involve computations on an indefinitely long data sequence. Therefore, we are only interested in MRDF graphs that can be executed in a nonterminating fashion. Consequently, we must be able to obtain schedules that can run infinitely using a finite amount of physical memory. Therefore, for our purposes, we say that an MRDF is correctly constructed if it can be scheduled periodically using a finite amount of memory. From now on, we will consider only well-constructed MRDF graphs.

The repetition vector for a correctly constructed MRDF graph with $|V|$ actors numbered 1 to $|V|$ is a column vector of length $|V|$. If each actor $v_{a}$ is fired a number of times equal to the ath entry of $r$, then the number of tokens per edge of the MRDF graph is equal to what it was in the initial state. Furthermore, $r$ is the smallest positive integer vector for which this property holds. The repetition vector $r$ is useful for generating infinite schedules for MRDF graphs. In addition, it will only exist if the MRDF graph has consistent sample rates (see [13]). The repetition vector can be computed in polynomial time [13].

An iteration of an MRDF graph is a sequence of actor firings such that each actor in the graph executes a number of times equal to its repetition vector entry.

\subsection{Single rate data flow}

An MRDF graph in which for every edge $e, \operatorname{prod}(e)=$ $\operatorname{cons}(e)=1$, is a single-rate data flow (SRDF) graph. Any MRDF graph can be converted into an equivalent SRDF graph. Each actor $i$ is replaced represented by $r(i)$ copies of itself, each representing a particular firing of the actor within each iteration of the graph. The input and output ports of these nodes are connected in such a way that the tokens produced and consumed by every firing of each actor in the SRDF graph remains identical to that in the MRDF graph (see [8]). SRDF graphs have very useful analytical properties.

For any given actor $i$ in the MRDF graph with an $r(i)$ entry in the repetition vector, if its copies in the equivalent SRDF graph are represented as $i_{p}, p=0,1, \ldots, r(i)-1$, the firing $k$ of $i_{p}$ corresponds to the firing $k \cdot r(i)+p$ of the original MRDF actor $a$. This fact will be used in the next section to establish a relation between SRDF and MRDF schedules.

The cycle mean of a cycle $c$ in an SRDF graph is defined as $\mu_{c}=\left(\sum_{i \in N(c)} t_{i} / \sum_{e \in E(c)} d_{e}\right)$, where $N(c)$ is the set of all nodes traversed by cycle $c$ and $E(c)$ is the set of all edges traversed by cycle $c$.

The maximum cycle mean (MCM) $\mu(G)$ of an SRDF graph $G$ is defined as

$$
\mu(G)=\max _{c \in C(G)} \frac{\sum_{i \in N(c)} t_{i}}{\sum_{e \in E(c)} d_{e}},
$$

where $C(G)$ is the set of simple cycles in graph $G$.

The MCM of an SRDF graph is closely related to its maximum attainable throughput. Many algorithms of polynomial 
complexity have been proposed to find the MCM (see [14] for an overview).

An MRDF is said to be first in first out (FIFO) if tokens cannot overtake each other in an actor. This means that between any two firings of the same actor, the first one to start is always the first one to produce outputs.

It is sufficient that an actor either has a constant execution time or belongs to a cycle with a single delay for the MRDF to have the FIFO property (see $[15,16]$ ). All MRDF models we consider in our work are FIFO. Moreover, if not stated otherwise, we will assume that every actor has an edge to itself with one delay on it, since most actors that represent tasks cannot execute self concurrently.

\section{TIMING PROPERTIES OF THE MODEL}

In this section, we discuss the relation between schedules that are a result of self-timed execution of data flow graphs and static periodic schedules. Some of this material is known from the literature $[12,17]$. The theorem about the relation between SPS and the MCM restates in a different form a result first published in [12]. The theorems concerning relations between ROSPS, SPS, and STS are, to the best of our knowledge, original contributions of this paper.

\subsection{Schedule notation}

The schedule function $s(i, k)$ represents the time at which the instance $k$ of actor $i$ is fired. The instance number is counted from 0 and, because of that, the instance $k$ corresponds to the $(k+1)$ th firing. Furthermore, we denote the finishing time of iteration $k$ of actor $i$ by $f(i, k)$ and the execution time of iteration $k$ of $i$ by $t(i, k)$. It always holds that $f(i, k)=s(i, j)+$ $t(i, k)$. If $t(i)$ is a WCET, then $t(i, k) \leq t(i)$, for all $k \in \mathbb{N}_{0}$.

\subsection{Admissible schedules}

A schedule is admissible if, for each actor in the graph, actor start times do not violate its firing rules. In [17] a theorem states a set of necessary and sufficient conditions for an admissible schedule, assuming constant execution times.

Theorem 1. A schedule $s$ is admissible if and only if for any arc $(i, j)$ in the graph,

$s(j, k) \geq s\left(i,\left\lceil\frac{(k+1) \cdot \operatorname{cons}(i, j)-d(i, j)-\operatorname{prod}(i, j)}{\operatorname{prod}(i, j)}\right\rceil\right)+t(i)$.

When applied to an SRDF graph, these equations become simply:

$$
s(j, k) \geq s(i, k-d(i, j))+t(i) .
$$

For an MRDF graph converted into SRDF for analysis purposes, a relation between the start times of the SRDF copies of an original MRDF actor can be established easily. Say that $a_{i}$ is the copy number $i$ of an MRDF actor $a$ in the equivalent SRDF graph. Then $s\left(a_{i}, k\right)=s(a, k \cdot r(a)+i)$.

From here on, scheduling will be discussed, for the sake of simplicity, on SRDF graphs.

\subsection{Self-timed schedules}

A self-timed schedule (STS), also known as an as-soon-aspossible schedule, of an SRDF graph is a schedule where each actor firing starts immediately if there are enough tokens in all its input edges.

The worst-case self-timed schedule (WCSTS) of an SRDF is the self-timed schedule of an SRDF where each actor always takes a time to execute equal to $t(i)$. The WCSTS of an SRDF graph is unique.

The WCSTS of an SRDF graph has an interesting property: after a transition phase of $K$ iterations, it will reach a periodic regime. The period is of $N(G) \cdot \mu(G)$ time units, where $N(G)$ is the cyclicity of the SRDF graph, as defined in [16]. $N(G)$ is equal to the minimum among the sums of delays of the critical cycles of the graph (see [16]).

The schedule for the periodic regime is

$$
s(i, k+N(G))=s(i, k)+N(G) \cdot \mu(G), \quad \forall k \geq K(G) .
$$

During periodic execution, $N(G)$ firings of $i$ happen in $N(G) \cdot \mu(G)$ time, yielding an average throughput [18] of $1 / \mu(G)$. For the transition phase, that is, for $k<K(G)$, the schedule can be derived by symbolic simulation given WCET of actors. Other known means of calculating $K(G)$ have the same exponential complexity, such as the one presented in [16].

\subsection{Static periodic schedules}

A static periodic schedule (SPS) of an SRDF graph is a schedule such that, for all nodes $i \in V$,

$$
s(i, k)=s(i, 0)+T \cdot k,
$$

where $T$ is the desired period of the SPS. The SPS can be represented uniquely by the values of $s(i, 0)$, for all $i \in V$.

Theorem 2. For any SRDF graph $G$, it is always possible to find an SPS schedule, as long as $T \geq \mu(G)$. If $T<\mu(G)$, then no SPS schedule exists.

Proof. Recall that according to (3) we know that every edge in the data flow graph imposes a precedence constraint of the form $s(j, k+d(i, j)) \geq s(i, k)+t(i)$ to any admissible schedule. Since the start times in an SPS schedule are given by (5), we can write for every edge $(i, j) \in E$ a constraint in the form

$$
\begin{gathered}
s(j, 0)+T \cdot(k+d(i, j)) \geq s(i, 0)+T \cdot k+t(i) \\
\Longleftrightarrow s(i, 0)-s(j, 0) \leq T \cdot d(i, j)-t(i) .
\end{gathered}
$$

These inequalities define a system of linear constraints. According to [19] this system has a solution if and only if the constraint graph does not contain any negative cycles for weights $w(i, j)=T \cdot d(i, j)-t(i)$.

The $\operatorname{MCM} \mu(G)$ is defined as

$$
\mu(G)=\max _{\forall c \in C(G)} \frac{\sum_{c} t(i)}{\sum_{c} d(i, j)}
$$


then, for each cycle $c \in C(G)$ it follows from the hypothesis that it must hold that

$$
T \geq \frac{\sum_{c} t(i)}{\sum_{c} d(i, j)} .
$$

The inequality (8) can be rewritten as

$$
\sum_{c}(T \cdot d(i, j)-t(i, j)) \geq 0
$$

that is, if $T \geq \mu(G)$, there are no negative cycles for weights $w(i, j)=T \cdot d(i, j)-t(i)$ and, therefore, the system given by (6) has at least one solution.

Therefore $1 / \mu(G)$ is the fastest possible rate (or throughput) of any actor in the SRDF. For an actor $a$ of MRDF graph $G$, it means that each one of its copies $a_{i}$ in the SRDF equivalent $G^{\prime}$ can execute at most once per $\mu(G)$. The fastest rate of $a$ is bounded by $r(a) \cdot 1 / \mu\left(G^{\prime}\right)$.

If an SPS has a period $T$ equal to the MCM of the SRDF graph $\mu(G)$, we say that this schedule is a rate-optimal static periodic schedule (ROSPS). Several SPSs for a given $G$ and $T$ can be found by solving the system of linear constraints given by (6).

A simple solution can be found for any given $T \geq \mu(G)$ by using a single-source shortest-path algorithm that can cope with negative weights, such as Bellman-Ford [8], but many other solutions may exist for any given graph and period. If an optimization criterion is specified that jointly maximizes and/or minimizes the start times for a set of chosen actors $S$, we get a linear programming (LP) formulation. Because of the particular structure of the problem, it can be solved efficiently using a min-cost-max-flow algorithm.

Notice that for an MRDF graph the SPS schedule of its SRDF equivalent specifies an independent periodic regime for each copy, but no periodicity is enforced between firings of different copies. If a strictly periodic regime with period $T / r(a)$ is required for actor $a$, extra linear constraints must be added to the problem. In some cases, this will result in an infeasible problem.

\subsection{Monotonicity}

We have already seen that it is possible to construct an SPS of any SRDF graph with a throughput equal to $1 / \mu(G)$ and that the WCSTS will eventually settle into a periodic behavior with an average throughput equal to $1 / \mu(G)$. Calculating $\mu(G)$ or trying to find an SPS schedule with period $\mu(G)$ are two ways to check for desired throughput feasibility. Two essential questions are yet to be answered: what happens during the transition phase, and how does STS behave with variable execution times? One property of SRDF graphs that allows us to give answers to these questions is monotonicity.

An SRDF $G$ with node valuation $t(i)$ is said to be monotonic if $t(i)$ can be replaced for any new valuation $t^{\prime}(i)$ such that $t^{\prime}(i) \leq t(i)$, for all $i \in V$, and any schedule $s(i, k)$ admissible for $t(i)$ is still admissible for $t^{\prime}(i)$.

The monotonic property is valid for SRDF graphs that have the FIFO property as described in the previous section.
For a proof of this, see [16]. From the monotonic property, we extract two very important relations.

\subsection{WCSTS and variable execution time STSs}

Because of monotonicity, if any given firing of an actor finishes execution faster than its worst-case execution time (WCET), then any subsequent events can never happen later than in the WCSTS, which can be seen as a function that bounds all start times in any execution of the graph with varying start times.

\subsection{Relation between the WCSTS and SPS}

Because of monotonicity, the start time of any actor cannot happen earlier than in the WCSTS: since in an STS firings happen as early as possible, there is no way to schedule anything earlier without violating the firing rule. As SPS schedules must assume worst-case execution times, the following theorem must hold.

Theorem 3. In any admissible SPS schedule of a graph $G=$ $(V, E)$, all start times can only be later or at the same time as in the WCSTS of that graph.

From this we draw an important conclusion: for a given graph, any SPS start time can be used as an upper bound to any start time of the same iteration of the same actor in the WCSTS.

\section{MODELING RESOURCE ALLOCATION}

In Sections 2 and 3, we stated properties of the data flow model without stating whether an actor, an edge, or a token represent in a real system. In this section, we describe the relation between the data flow model and the system and show how we can include design-time scheduling decisions and the effects of run-time scheduling in the data flow model.

\subsection{Task graphs}

The MRDF that serves as an input to the resource allocation process is a functional description of the job where every actor corresponds to a computational task. Because of this, we call such a graph the task graph of the application. At this stage, the execution times of actors correspond to the WCETs of tasks on a specific processor type and executing in isolation (i.e., with no interferences are taken into account). As resource allocation decisions are taken, the graph becomes more implementation aware. Communication through the network is modeled, buffers are bounded, the execution times of actors that represent tasks include the effects of local scheduling. Note that we make a strong distinction between the execution time of a task and the execution time of an actor. The execution time of a task is the time interval between the moment when the actor that represents the task starts a firing and finishes it, when processing resources are dedicated, that is, neither pre-emption nor any other sort of interference can occur. The execution time of an actor may take into account such effects, as we describe 
below. We will now list some of the resource allocation decisions and how they can be modeled.

\subsection{Buffer capacity}

A buffer capacity constraint can be expressed in MRDF as a back-edge from the consumer of a FIFO to its producer. As the number of tokens in the cycle between producer and consumer can never exceed the number of initial tokens in that cycle, the edge that models the actual data FIFO can never have more than the number of tokens initially placed in the "credits" back edge. This also means that an actor cannot be fired without enough space being available in each of its output FIFOs, which represents the worst-case effect of backpressure.

\subsection{Communication channels}

Depending on the target architecture and the level of detail required, communication channels might be modeled in different ways. In $[15,20]$ models are derived for the Æthereal network. Many different models for the same network are possible, depending on the level of abstraction. The simplest one is used in this paper, for the sake of simplicity. The reader is encouraged to consult $[15,20]$ to find more precise and detailed models of the Æthereal network that our tools use. Any of these models is parametric. In our simple model only the time between consecutive token transmissions, the $t$ valuation of the communication actor must be set by the designer.

\subsection{Task scheduling}

Modeling task scheduling only applies to actors that represent tasks. There are two types of task-scheduling mechanisms that we may be interested in modeling: compile-time and run-time scheduling.

Compile-time scheduling (CTS) encompasses scheduling decisions that are fixed at compile time, such as static order scheduling. If two tasks running at the same rate are mapped onto the same processor, with a static order per iteration, an arc with 0 delay added from the first to the second conveniently models the dependency. Several actors can be chained this way. This also works for static schedules. An edge from the last actor in the static order to the first with 1 delay models the fact that all the actors in the static schedule chain are now mutually exclusive (since they share a processor). This can only be done between tasks that execute at the same rate (i.e., have the same value in their respective repetition vector entry).

Run time scheduling (RTS) cannot be resolved at compiletime, because it depends on the run-time task-to-processor assignment, which in turn depends on the dynamic job mix. It is handled by the local scheduling mechanism, or dispatcher. Modeling the effects of the dispatcher is needed to include in the compile-time analysis the effects of sharing processing resources among jobs. If the WCET of the task, the settings of the local dispatcher, and the amount of computing resources to be given to the task are known, then the actor execution time can be set to reflect the worst-case response time of that task running in that local dispatcher, with that particular amount of allocated resources. In [21], we show how this can be computed for a TDMA scheduler and, in [5], for a non-preemptive round-robin.

If the amount of computing resources to be given to the task is not known, it must be found. A relative deadlinethe maximum time that it can take in the implementation between actor enabling and the end of its the executioncan be attributed to the task by taking the end-to-end timing requirements of the job and whatever knowledge we have about the WCETs of the tasks in this job. Essentially, the problem amounts to choosing how much time each task can take to execute, given that it must at least take as much time as its WCET, and that the end-to-end temporal requirements must be met. The relative deadline can then be used to infer the resource budget for the task, given local dispatcher settings.

\section{INTERFACING WITH THE ENVIRONMENT}

The input of many systems is provided by a strictly periodic source like an A/D converter and the output data is often supplied to a strictly periodic sink like a D/A converter. In some cases, there is a maximum latency constraint specified relatively to the source. In other systems, bursts of data are received in the form of packets. With the analysis techniques that are presented in this section we can derive whether the environment can impose periodic/sporadic/bursty execution of a source or sink without causing a violation of latency constraints and compute bounds to the maximum latency relatively to the source.

\subsection{Strictly periodic actors within a self-timed schedule}

There are situations where it is essential to guarantee that an actor has a strictly periodic behavior. For instances an audio output sink should not experience any hiccups due to the aperiodic behavior caused by either the initial transition phase of the STS or by the variation of execution times from iteration to iteration. Moreover, we want to be able to compose functionality by feeding the output of a job as the source to another job. This is greatly simplified if jobs can see each other as periodic sources or sinks, as no joint analysis will be required.

We have already established that for any given period $T \geq$ $\mu(G)$, it is possible to generate an SPS such that all actors are strictly periodic. On the other hand, we know that in an STS start times can only be equal or earlier than in an SPS with the same period, that is,

$$
s_{\mathrm{STS}}(i, k) \leq s_{\mathrm{SPS}}(i, k)=s_{\mathrm{SPS}}(i, 0)+T \cdot k .
$$

Assume that we will force only a minimum time interval of $T$ between successive starts of an SRDF actor by introducing an additional actor $q$ (see Figure 2 ) with execution time $t(q)=T-t(i)$, then

$$
s(i, k) \geq s(i, k-1)+T \Longrightarrow s(i, k) \geq s(i, 0)+T \cdot k .
$$




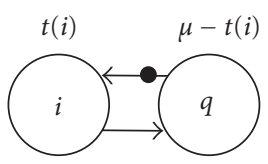

Figure 2: Actor $q$ will enforce a minimum interval $\mu(G)$ between successive firings of actor $i$.

From (10) and (11) it follows that

$$
s(i, 0)+T \cdot k \leq s_{\mathrm{STS}}(i, k) \leq s_{\mathrm{SPS}}(i, 0)+T \cdot k
$$

because we can set

$$
s_{\mathrm{SPS}}(i, 0)=s(i, 0)
$$

and we conclude that

$$
s(i, k)=s(i, 0)+T \cdot k .
$$

What does this imply? That if we fix the start time of its first firing such that the condition in (13) holds for at least one ROSPS of $G$, we can guarantee $i$ to execute in a strictly periodic fashion, independently of any timing variations that occur in the rest of the graph. We do not have to enforce a strict initial start time, but guarantee that $s(i, 0)$ is equal to any of the admissible $s_{\text {ROSPS }}(i, 0)$. This means that $s(i, 0)$ must be between its earliest and latest start times in admissible ROSPS schedules - any value in this interval is valid since linear programs have a convex solution space. These earliest and latest start times can be computed by finding two ROSPS via LP formulations: one that minimizes i's start time, and another one that maximizes it.

In the implementation, actor $i$ must wait for a time equal to the computed minimum $s(i, 0)$ before firing the first time. After this, the actor may need a local timer that enables its execution every $T$ units of time, and releases outputs of the previous iteration, such that it exhibits a constant execution time. Essentially, we statically schedule one actor, allowing the rest of the system to continue to be self timed.

\subsection{Latency analysis}

\subsubsection{Definition of latency and maximum latency}

Latency is the time interval between two events. We measure latency as the difference between the start times of two specific firings of two actors, that is,

$$
L(i, k, j, p)=s(j, p)-s(i, k)
$$

where $i$ and $j$ are actors, $p$ and $k$ are iterations. We say that $i$ is the source of the latency constraint, and $j$ is the sink.

Typically, we are interested in cyclic latency requirements, such that we can define that between the $p$ th firing of actor $i$ in any given iteration $k$ and the $q$ th firing of $j$ in iteration $k+n$, where $n$ is a fixed iteration distance, a maximum latency limit is preserved:

$$
\begin{aligned}
\widehat{L}(i, p, j, q, n) & =\max _{k \geq 0} L(i, r(i) \cdot k+p, j, r(j) \cdot(k+n)+q) \\
& =\max _{k \geq 0}(s(j, r(j) \cdot(k+n)+q)-s(i, r(i) \cdot k+p))
\end{aligned}
$$

with $0 \leq p \leq r(i)$ and $0 \leq q \leq r(j)$.

In order to make the following discussion simpler, we will restrict it to SRDF graphs, where the $p$ and $q$ firing numbers relative to the start of iteration can be omitted since they are always equal to 0 :

$$
\widehat{L}(i, j, n)=\max _{k \geq 0} L(i, k, j, k+n)=\max _{k \geq 0}(s(j, k+n)-s(i, k)) .
$$

Notice that any latency constraint of the type of (16) can be converted directly into a constraint of the type of (17) in the SRDF equivalent graph, by applying the relation between MRDF actors and their SRDF copies.

Self-timed scheduling with variable execution times makes latency analysis difficult. The problem is that while it is easy to find an upper bound for $s(j, k+n)$ using the relations between STS, WCSTS, and SPS that we developed in Section 3, it is still difficult to find a lower bound for $s(i, k)$. In many cases, however, the best-case execution time of the source can be inferred. The simplest case happens if the job has a strictly periodic source. We will start by analysing that case.

\subsubsection{Maximum latency from a periodic source}

The start times of a periodic source are given by

$$
s(i, k)=s(i, 0)+\mu(G) \cdot k
$$

Note that the earliest possible value of $s(i, 0)$ is given by the WCSTS of the first iteration. Because of Theorem 3, the start times of $j$ executing in STS are bounded by the start time of any ROSPS schedule, that is,

$$
s(j, k+n) \leq \check{s}_{\text {ROSPS }}(j, 0)+\mu(G) \cdot(k+n),
$$

where $\check{s}_{\text {ROSPS }}(j, 0)$ represents the smallest $s(j, 0)$ in an admissible ROSPS. Equation (18) gives us an exact value of $s(i, k)$, while (19) gives us an upper bound on $s(j, k+n)$. By taking the upper bound for $s(j, k+n)$ and the lower bound for $s(i, k)$, we get

$$
\begin{aligned}
\hat{L}(i, j, n) & =\max _{k \geq 0}(s(j, k+n)-s(i, k)) \\
& \leq \check{s}_{\text {ROSPS }}(j, 0)-s(i, 0)+\mu(G) \cdot n .
\end{aligned}
$$

Therefore, we can determine the maximum latency from a periodic source just by calculating an ROSPS with the earliest start time $j$ and a WCSTS for the earliest start time of $i$. 


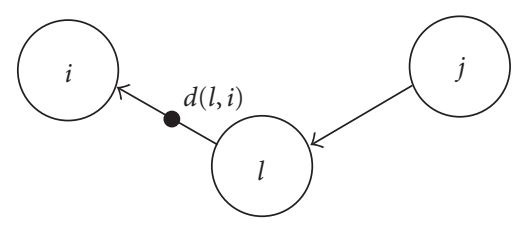

FIGURE 3: Modeling a latency constraint in an SRDF graph.

\subsubsection{Modeling latency constraints from a periodic source}

We can also represent the latency constraint in terms of the throughput constraint. This is useful when employing an MCM algorithm to check for constraint violation. We add to the graph an actor $l$ with constant execution time $t(l)$ and an edge $(j, l)$ and an edge $(l, i)$ with $d(l, i) \geq 1$ as shown in Figure 3 . The actor $l$ does not have a self edge. The period of the source actor $i$ is $\mu(G)$.

Modeling a latency constraint in this way is only possible between actors with equal repetition vector entries, since we cannot have arcs between specific firings of actors. However, if such model is required, one can always convert the MRDF graph onto its equivalent SRDF.

It holds that

$$
s(i, k+d(l, i)) \geq s(j, k)+t(l) .
$$

Please notice that $t(j)$ is not added to the right-hand side since we are looking for a lower bound and the best execution time of $j$ is only bounded from below by 0 . If a higher best execution time is known for $t(j)$, it should be added here.

By replacing (5) in (21) we obtain

$$
\begin{aligned}
s(j, k) & -s(i, k) \leq \mu(G) \cdot d(l, i)-t(l) \\
& \Longleftrightarrow \widehat{L}(i, j, 0) \leq \mu(G) \cdot d(l, i)-t(l) .
\end{aligned}
$$

By setting adequately the values of $t(l)$ and $d(l, i)$ we effectively model a latency constraint in terms of the throughput, that is, an infringement of the latency constraint will be detected as an increase of $\mu(G)$, that is, an infringement of the minimum throughput constraint. The parameters can be set for any values of $d(l, i)$ and $t(l)=\mu \cdot d(l, i)-L$, as long as $t(l), d(l, i) \geq 0$. The construct $l,(j, l),(l, i)$ does not need to have any equivalent in the implementation.

\subsubsection{Maximum latency from a sporadic source}

In reactive systems, it is frequent that the source is not strictly periodic, but produces tokens sporadically, with a minimal time interval $\mu(G)$ between subsequent firings. Typically, a maximum latency constraint must be guaranteed. This is the case in the WLAN receiver we show in the case studies section. It is easy to see that the MRDF has to support a throughput of $1 / \mu(G)$ in order to guarantee that it cannot be overran by the source. In this section, we derive the maximum latency relative to a sporadic source. First, we define a sporadic source more formally.
Definition 1. A source is sporadic if $s(i, k) \geq s(i, k-1)+\mu(G)$.

We introduce a strictly periodic schedule of source $i$ with period $\mu(G)$, that is,

$$
s^{\prime}(i, k)=s^{\prime}(i, 0)+\mu(G) \cdot k .
$$

We define $\delta(k)$ as

$$
\delta(k)=s(i, k)-s^{\prime}(i, k) .
$$

Lemma 1. If a source is sporadic, then

$$
\delta(k+1)-\delta(k) \geq 0 .
$$

Proof. We replace the definition of $\delta$ in (25):

$$
\left(s(i, k+1)-s^{\prime}(i, k+1)\right)-\left(s(i, k)-s^{\prime}(i, k)\right) \geq 0 .
$$

As $s^{\prime}(i, k+1)=s^{\prime}(i, k)+\mu(G),(26)$ becomes

$$
s(i, k+1)-s(i, k) \geq \mu(G)
$$

which is true by hypothesis, since our source is sporadic.

Lemma 2. The maximum value of $m$, for which increasing the start of iteration $k$ of actor $i$ has no effect on the start time of iteration $q$ of actor $j$, with $q<k+m$, can be computed in polynomial time.

Proof. For each edge $(g, h)$ in an admissible schedule it holds that

$$
s(h, k+d(g, h)) \geq s(g, k)+t(g) .
$$

If we assume that $g$ and $h$ execute strictly periodically and $t(g)=0$, we can rewrite (28) in the following form:

$$
s(h, 0)+\mu(G) \cdot(k+d(g, h)) \geq s(g, 0)+\mu(G) \cdot k .
$$

The number of firings of an actor $f$ at time $t$ in terms $s(f, 0)$ is equal to

$$
n(f, t)=\frac{t-s(f, 0)}{\mu(G)} .
$$

Given (30) we can rewrite (29) as

$$
-n(h, t) \cdot \mu(G)+t+\mu(G) \cdot d(g, h) \geq-n(g, t) \cdot \mu(G)+t .
$$

This is equivalent to

$$
n(h, t) \leq n(g, t)+d(g, h) .
$$

We want to find how many times we can execute $j$ more than $i$ while respecting the firing rules of the actors. This number of iterations that $j$ can execute at any point in time 
more than $i$ must be the number of iterations of $j$ that are independent of $i$. We can find the maximum iteration distance $m=n(j, t)-n(i, t)$ with a single-source shortest-path algorithm such at Bellman-Ford that takes (32) for every edge as a constraint and implicitly maximizes the iteration distance. A solution of the shortest path problem that respects for each edge (32) indicates that a schedule exists in which the iteration distance is $m$. We conclude that the maximum iteration distance for every schedule is $m$ because the existence of a schedule that results in an iteration distance does not depend on the execution time of the actors nor on the start times of the actors. Because the iteration distance is defined for any point in time we conclude that execution $k$ of $i$ does not have an effect on execution $q$ of $j$ with $q<k+m$.

Theorem 4. If in a schedule all start times are self timed, except for an actor $i$, which is delayed during the first $k$ firings with at most $\delta(k) \geq 0$, that is, $s(i, k) \leq s^{\prime}(i, k)+\delta(k)$ then, for $m$ according to Lemma 2 and $p \leq k+m$, the start time of another actor $j$ is bounded by $s(j, p) \leq s^{\prime}(j, p)+\delta(k)$, with $s^{\prime}(i, k)=$ $s^{\prime}(i, 0)+\mu(G) \cdot k$ and $s^{\prime}(i, 0)=s(i, 0)$ and with $s^{\prime}(j, k)$ the start times of $j$ if $s^{\prime}(i, k)$ is used as source.

Proof. If input tokens of an actor are delayed by at most $\delta(k)$ then an output token of this actor is delayed by at most $\delta(k)$. Thus, if $y_{n}=\max \left(x_{1}+\delta_{1}, x_{2}+\delta_{2}, \ldots, x_{n}+\delta_{n}\right)$, then $y_{n} \leq \max \left(x_{1}, x_{2}, \ldots, x_{n}\right)+\delta(k)$ with $\delta_{i} \leq \delta(k)$. The output tokens of one actor are the input tokens of another actor. If the input tokens of all actors are delayed by at most $\delta(k)$ then the production of output tokens is also delayed by at most $\delta(k)$. Lemma 2 implies that $s(j, p)$ with $p<k+m$ is not affected by the value of $\delta(q)$ with $q>k$. Therefore, we conclude that if $s(i, k) \leq s^{\prime}(i, k)+\delta(k)$ then for $p \leq k+m$, $s(j, p) \leq s^{\prime}(j, p)+\delta(k)$.

Theorem 5. The latency between the kth start of a sporadic source actor $i$, that is, $s(i, k)$, and the $(k+n)$ th firing of actor $j$, that is, $s(j, k+n)$ with $n<m$ and $m$ according to Lemma 2, is at most $\check{s}_{\text {ROSPS }}^{\prime}(j, 0)-s^{\prime}(i, 0)+\mu(G) \cdot n$ with $s^{\prime}(i, k)=s^{\prime}(i, 0)+$ $\mu(G) \cdot k$ and $s^{\prime}(i, 0)=s(i, 0)$.

Proof. The start time of actor $i$ relative to the start of a strict periodic actor $i$ is

$$
s(i, k)=s^{\prime}(i, k)+\delta(k) .
$$

We define $s^{\prime}(j, k)$ as the start times of $j$ if $s^{\prime}(i, k)$ is used as source.

It follows directly from Lemma 1 that $\max _{p \leq k} \delta(p)=$ $\delta(k)$. Given (33) and $n<m$ it follows from Theorem 4 that

$$
s(j, k+n) \leq s^{\prime}(j, k+n)+\delta(k) .
$$

We know that the maximum start time in an STS is not later than the earliest possible start time in an ROSPS, that is,

$$
s^{\prime}(j, k+n) \leq \check{s}_{\mathrm{ROSPS}}^{\prime}(j, k+n) .
$$

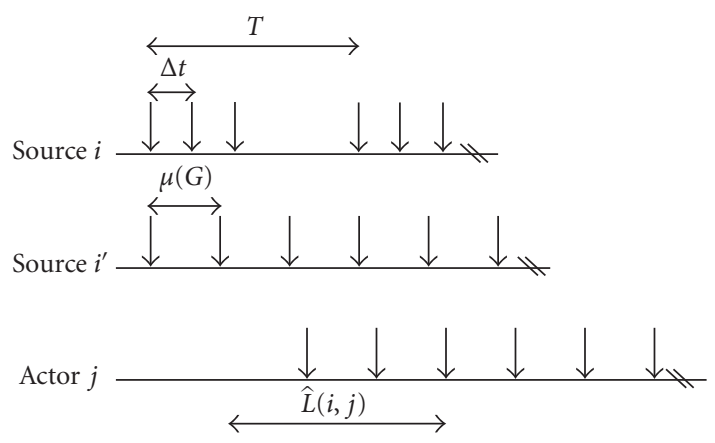

FIgURE 4: Arrival times of tokens of a bursty source relatively to strictly periodic source.

Given (35) and by definition of ROSPS, we can rewrite (34) in the following form:

$$
s(j, k+n) \leq \check{s}_{\mathrm{ROSPS}}^{\prime}(j, 0)+\mu(G) \cdot(k+n)+\delta(k) .
$$

Therefore, the maximum latency is bounded by

$$
\begin{aligned}
\widehat{L}(i, j, n)= & \max _{k \geq 0}(s(j, k+n)-s(i, k)) \\
\leq & \check{s}_{\mathrm{ROSPS}}^{\prime}(j, 0)+\mu(G) \cdot(k+n)+\delta(k) \\
& -s^{\prime}(i, 0)-\mu(G) \cdot k-\delta(k) \\
\leq & \check{s}_{\mathrm{ROSPS}}^{\prime}(j, 0)-s(i, 0)+\mu(G) \cdot n .
\end{aligned}
$$

The latency $\hat{L}(i, j, n)$ is not defined for $n>m$ because the start time of execution $k+n$ of $j$ is dependent on the start time of execution $k+1$ of $i$. However, the maximum difference between $s(i, k+1)$ and $s(i, k)$ is undefined for a sporadic source.

When defined, the latency $\hat{L}(i, j, n)$ with a sporadic source has the same upper bound as the latency for the same source, sink, and iteration distance in the same graph with a periodic source.

\subsubsection{Maximum latency from a bursty source}

We characterize a bursty source as a source that may fire at most $n$ times within a $T$ time interval, with a minimal $\Delta t$ interval between consecutive firings. A job that processes such a source must have $\mu(G) \leq T / n$ to be able to guarantee its processing within bounded buffer space. Moreover, if $\mu(G) \leq \Delta t$, then we are in the presence of the previous case, that is, maximum latency from a sporadic source. If $\mu(G) \geq \Delta t$ then latency may accumulate over iterations, as the job processes input tokens more slowly than they arrive. The maximum latency must occur when the longest burst occurs, with the minimum interval between firings of the source, that is, a burst of $n$ tokens with $\Delta t$ spacing. Because of monotonicity, making the source execute faster cannot make the sink execute slower, but it also cannot force it to execute faster. Therefore we can compute the latency as shown in Figure 4.

As depicted in Figure 4, the tokens of the bursty source $i$ will arrive earlier than the periodic source $i^{\prime}$. Therefore, while 
iteration $n-1$ after the beginning of the burst (iteration 0 ) happens the earliest time $s(i, n-1)=s_{\text {ROSPS }}(i, 0)+(n-1) \cdot \Delta t$. The iteration $n-1$ of $j$ happens the latest at $s(j, n-1) \leq$ $\check{s}_{\text {ROSPS }}(j, 0)+(n-1) \cdot \mu(G)$. Therefore, a bound on the maximum latency is given by

$$
\widehat{L}(i, j) \leq \check{s}_{\text {ROSPS }}(j, 0)-s_{\text {ROSPS }}(i, 0)+(n-1)(\mu(G)-\Delta t) .
$$

\section{CASE STUDIES}

Two case studies are presented in this section. These case studies illustrate the mapping of a job to a multiprocessor architecture and the a model for a job with a maximum latency requirement and a sporadic source.

\subsection{Simplified channel equalizer}

In this section, we illustrate the mapping of a channel equalizer job onto the architecture in Figure 1. This channel equalizer has a strict periodic source and sink. The maximum latency between source and sink is not specified.

The SRDF graph of the channel equalizer is shown in Figure 5. Actors A to D run on EPICS cores. The FIR1 and FIR2 actors run on FIR accelerators. The source of the channel decoder is the A/D and the sink is an actor that executed strictly periodically. The source is periodic with a frequency of $325 \mathrm{KHz}$. The EPICS cores run at a speed of $125 \mathrm{MHz}$ and therefore, in EPICS cycles, we get a required MCM of $\mu(G)=385 \geq 125 \mathrm{M} / 325 \mathrm{~K}$ cycles.

Both the A/D source and the D/A source have their buffer sizes limited to 4 tokens because that is the size of output/input queues on the network interface of the peripherals subsystem. This is a hardware constraint. For all other NIs, the maximum size is substantially larger, and can be assumed to be large enough for the purposes of this example.

The cycle B-FIR1-C-D-FIR2 can become critical as a result of network communication latency, since its cycle-mean $\left(\mu_{c}\right)$ is $5 * 70 / 1=350$ cycles, which means that only 35 cycles in total are available for communication. It is an architecture limitation that the lowest latency communication channels in the network have a latency of 8 cycles for tokens with a size of 2 words. If we insert the network nodes, as in Figure 6, we get that now $\mu_{c}=5 * 70+4 * 8=382$, which is just below the required MCM. Figure 6 also represents the maximum buffer size for the source output and sink input by inserting back edges from consumers to producers and decisions about static ordering scheduling of actors in processor as described in the figure.

Actors B, C and D must be statically ordered because additional delay due to run-time arbitration would result in a cycle mean that is larger than the MCM. It is decided that they share a processor: since they are already mutually exclusive (because they all belong to the same 1 token cycle), only one is enabled at a time, and therefore none of them may delay the execution of another. The two FIR actors are also made to share an FIR accelerator. The static order of these actors does add several more cycles to the graph, but these cycles have clearly lower cycle means than the B-FIR1-C-DFIR2 cycle, and therefore never become critical.

As communication to and from A does not add a critical cycle, $N 1$ and $N 2$ can be rather slow communication nodes. In fact, they only need to communicate a single word every $\mu(G)$ cycle. They are therefore set to $t(N 1)=t(N 2)=385$ cycles.

Most decisions are now taken, except for buffer sizing and strictly periodic behavior of the sink. We check how late the first activation of the sink must happen so that the inputs are always ready on time. By computing two SPSs from linear program formulations (minimize start of sink, maximize start of sink), we determine that the first activation of the sink must happen between cycle 2151 and cycle 2921 .

The lower limit on the start of the sink is necessary because the first token may be available much earlier than the worst case of the propagation through the graph since it may happen, for instance, that the best-case execution happens jointly to $\mathrm{A}, \mathrm{B}$, and $\mathrm{C}$ in the first iteration and the second iteration takes worst-case time. If the sink actor is executed as soon as possible on the first iteration, it may take a much longer time than $\mu(G)$ before its second activation. The upper limit on the start is caused by the fact that the sink must free space in the buffer before the 5th firing of the N5 actor may occur. Failure to do this may cause a backpressure domino effect that delays the whole execution. We thus set a timer activated by the beginning of the first firing of the source that only allows sink execution 2151 cycles later.

Given the computed setting, we can calculate a minimal buffer sizing, with any of the techniques described in [8] or [22] (based on static scheduling).

\subsection{Wireless $L A N$ receiver}

In this section, we illustrate the modeling and analysis of a wireless LAN receiver application that has a sporadic source and a maximum latency constraint. The source is sporadic because only a minimum distance between the arrivals of frames with data is defined.

The timing requirement of a WLAN receiver is a maximum latency between the beginning of the transmission of the frame and the emission of an acknowledge message by the receiver, which must happen a fixed time (the SIFS time) after the end of the transmission.

In Figure 7 we show the timing of the frame transmission and a simplified state machine specification of what the receiver must do. Each frame is received symbol by symbol. First, a fixed number of synchronization symbols are sent, this sequence has a constant length of $S$ symbols. While these synchronization symbols are received, the receiver must detect the beginning of the frame and start a synchronization procedure that needs at least two symbols to be done. Every time the receiver fails to either detect or synchronize, it must start detection again. After the synchronization sequence, the frame starts transmitting a fixed-size header of $H$ symbols, which must be decoded to determine the size of the payload. After the header, the variable-sized payload is transmitted. 


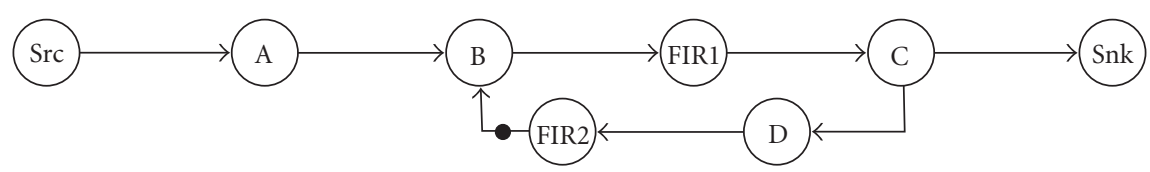

FIGURE 5: Implementation-unaware SRDF graph of a simplified channel equalizer.

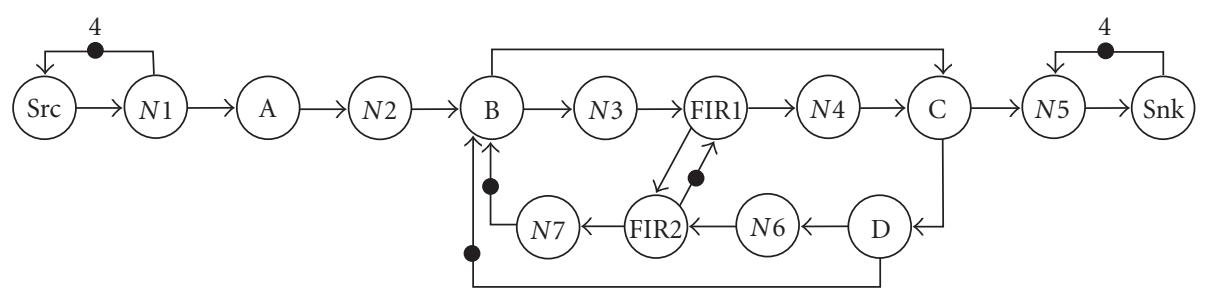

FIGURE 6: Implementation-aware SRDF graph of a simplified channel equalizer.

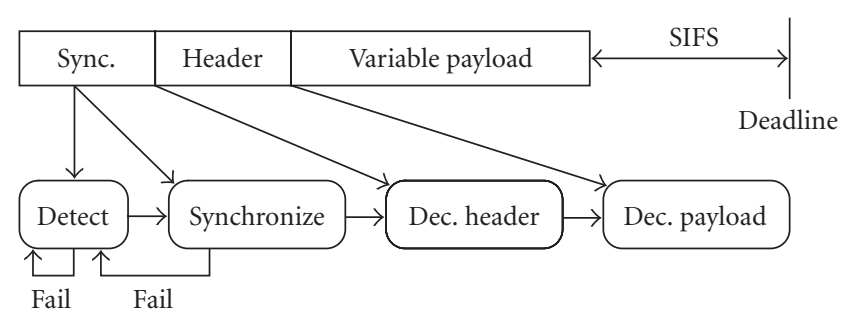

Figure 7: Real-time requirements and state diagram for $902.1 \mathrm{~b}$ (WLAN) reception job.

The actual deadline for finishing payload decoding is the end of the frame plus the so-called SIFS timing.

We model this application in MRDF by first realizing that the only case where the timing actually matters is when both detection and synchronization succeed. We also see that the worst case of successful synchronization happens when the symbol where synchronization is achieved is the one right before the beginning of the header, which means that detection must have happened in the previous symbol.

The simplified MRDF model is shown in Figure 8, where the complexity of the task graph for each one of the stages is hidden in a single actor. We model the source as a chain of constant execution time actors $\mathrm{Rx}$, each one corresponding to one of the symbols, except the first $S-2$ symbol productions which are modeled as a single actor Rx1 with the same execution time as the other Rx nodes. Like this, we are able to explicitly express that different tasks are activated by the arrival of particular symbols within the packet. In addition, the two small actors without caption are only used to make rate conversion and take no execution time: after $H$ tokens are produced by $H$ executions of the third $\mathrm{Rx}$, the conversion actor produces $N$ tokens necessary for the $N$ copies of the 4th Rx actor, which corresponds to the reception of $N$ payload symbols. This is just a modeling trick to make the graph shorter than an SRDF representation where a source would be needed for each symbol in the frame.

Our MRDF model is parametric, in that the number of symbols $N$ that constitute the payload is variable. As the maximum size is well defined, we can, if needed, make a separate analysis for each message size, or rely on the observation that the worst case either happens when $N=1$, since the receiver has much less time to catch up, and it is not possible to pipeline payload decoding, or when $N$ is maximum, if the payload decoding stage has a lower throughput than needed.

The maximum latency requirement is modeled by adding an actor (labelled SIFS) where $t$ (SIFS) is the SIFS timing to the source actor chain, and closing it as a loop. We now also direct an edge from the end of the decoder block to the first source. The source starts every $D=t(\mathrm{Rx}) *(S+H+$ $N)+t($ SIFS). This is equivalent to the deadline shown in Figure 7 . What we did was essentially according to the model for a maximum latency constraint presented in Section 5: we added a path from the decoder actor (the sink of the latency requirement) to the first $\mathrm{Rx}$ (the source of the latency requirement). We made $t(l)=0$ and $d(l, \mathrm{Rx} 1)=1$, which means that the enforced latency is $L \leq \mu(G) \cdot d_{i j}=D$, as intended. If any cycle during implementation becomes longer than $D$, this will be detected as an MCM constraint violation.

\section{CONCLUSION}

We developed analysis methods based on the monotonic property of MRDF graphs and especially on the relation between self-timed and periodic schedules. We use this relation to reason not only about average throughput- to which analysis of self-timed schedules of MRDF graphs traditionally limits itself-but also about maximum latency and interfacing with the environment. Interfacing with the environment includes the use of sources with a strictly periodic behavior but also an aperiodic or bursty behavior which requires knowledge about the temporal behavior during the 


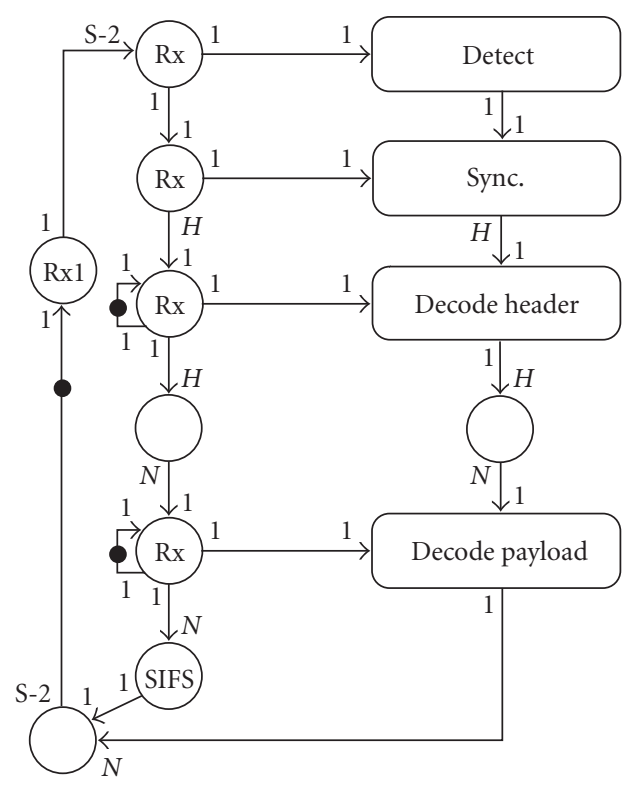

Figure 8: MRDF model of 902.1b (WLAN) reception job.

transition phase of the system and not only about the temporal behavior during the steady state of the system.

These methods allow an iterative mapping process, where every implementation decision becomes a constraint in the implementation-aware MRDF model in the next iteration. At any iteration this MRDF model can be used for verifying that temporal constraints are still met after each design decision is taken. It also provides a rationale for preferring certain decisions to others.

The presented methods are used for the programming of a software-defined radio and a car infotainment system. Two examples illustrate the usage of our methods: a channel equalizer and a WLAN receiver. The first example illustrates the design flow for a system with a strictly periodic source and the second example illustrates the analysis of a system with a sporadic source and a maximum latency constraint.

\section{REFERENCES}

[1] G. C. Buttazzo, Hard Real-Time Computing Systems, Kluwer Academic Publishers, Boston, Mass, USA, 1997.

[2] A. J. M. Moonen, R. van den Berg, M. J. G. Bekooij, H. Bhullar, and J. van Meerbergen, "A multi-core architecture for in-car digital entertainment," in Proceedings of Global Signal Processing Conference \& Expos for the Industry, Santa Clara, Calif, USA, October 2005.

[3] K. Goossens, J. Dielissen, and A. Rădulescu, "Æthereal network on chip: concepts, architectures, and implementations," IEEE Design and Test of Computers, vol. 22, no. 5, pp. 414-421, 2005.

[4] A. Hansson, K. Goossens, and A. Rădulescu, "A unified approach to constrained mapping and routing on networkon-chip architectures," in Proceedings of International Conference on Hardware/Software Codesign and System Synthesis (CODES+ISSS '05), pp. 75-80, Jersey City, NJ, USA, September 2005.
[5] O. M. Moreira, J. D. Mol, M. J. G. Bekooij, and J. van Meerbergen, "Multiprocessor resource allocation for hard-real-time streaming with a dynamic job-mix," in Proceedings of IEEE Real-Time and Embedded Technology and Applications Symposium (RTAS '05), pp. 332-341, Francisco, Calif, USA, March 2005.

[6] O. M. Moreira, M. J. G. Bekooij, and J. D. Mol, "Online resource mangement in a multiprocessor with a network-onchip," in Proceedings of the 22nd Annual ACM Symposium on Applied Computing (SAC '07), Seoul, Korea, March 2007.

[7] E. A. Lee and D. G. Messerschmitt, "Synchronous data flow," Proceedings of the IEEE, vol. 75, no. 9, pp. 1235-1245, 1987.

[8] S. Sriram and S. S. Bhattacharyya, Embedded Multiprocessors: Scheduling and Synchronization, Marcel Dekker, New York, NY, USA, 2000.

[9] N. Bambha, V. Kianzad, M. Khandelia, and S. S. Bhattacharyya, "Intermediate representations for design automation of multiprocessor DSP systems," Design Automation for Embedded Systems, vol. 7, no. 4, pp. 307-323, 2002.

[10] S. Goddard and K. Jeffay, "Managing latency and buffer requirements in processing graph chains," The Computer Journal, vol. 44, no. 6, pp. 486-503, 2001.

[11] M. Jersak, K. Richter, and R. Ernst, "Performance analysis of complex embedded systems," International Journal of Embedded Systems, vol. 1, no. 1-2, pp. 33-49, 2005.

[12] R. Reiter, "Scheduling parallel computations," Journal of the ACM, vol. 15, no. 4, pp. 590-599, 1968.

[13] E. A. Lee and D. G. Messerschmitt, "Static scheduling of synchronous data flow programs for digital signal processing," IEEE Transactions on Computers, vol. 36, no. 1, pp. 24-35, 1987.

[14] A. Dasdan, "Experimental analysis of the fastest optimum cycle ratio and mean algorithms," ACM Transactions on Design Automation of Electronic Systems, vol. 9, no. 4, pp. 385-418, 2004.

[15] P. Poplavko, T. Basten, M. J. G. Bekooij, J. van Meerbergen, and B. Mesman, "Task-level timing models for guaranteed performance in multiprocessor networks-on-chip," in Proceedings of International Conference on Compilers, Architecture, and Synthesis for Embedded Systems (CASES '03), pp. 63-72, San Jose, Calif, USA, October-November 2003.

[16] F. Baccelli, G. Cohen, G. Olsder, and J.-P. Quadrat, Synchronization and Linearity, John Wiley \& Sons, New York, NY, USA, 1992.

[17] R. Govindarajan and G. R. Gao, "A novel framework for multirate scheduling in DSP applications," in Proceedings of International Conference on Application-Specific Array Processors, pp. 77-88, Venice, Italy, October 1993.

[18] A. H. Ghamarian, M. C. W. Geilen, S. Stuijk, et al., "Throughput analysis of synchronous data flow graphs," in Proceedings of the 6th International Conference on Application of Concurrency to System Design (ACSD '06), pp. 25-36, Turku, Finland, June 2006.

[19] T. H. Corman, C. E. Leiserson, R. L. Rivest, and C. Stein, Introduction to Algorithms, McGraw-Hill, New York, NY, USA, 2001.

[20] A. J. M. Moonen, M. J. G. Bekooij, and J. van Meerbergen, "Timing analysis model for network based multiprocessor systems," in Proceedings of the 15th Annual Workshop of Circuits, System and Signal Processing (ProRISC '04), pp. 91-99, Veldhoven, The Netherlands, November 2004. 
[21] M. J. G. Bekooij, R. Hoes, O. M. Moreira, et al., "Dataflow analysis for real-time embedded multiprocessor system design," in Dynamic and Robust Streaming in and between Connected Consumer Electronic Devices, vol. 3, pp. 81-108, Springer, New York, NY, USA, 2005.

[22] R. Govindarajan, G. R. Gao, and P. Desai, "Minimizing memory requirements in rate-optimal schedules," in Proceedings of International Conference on Application Specific Array Processors, pp. 75-86, San Francisco, Calif, USA, August 1994.

Orlando M. Moreira graduated from the University of Aveiro in Portugal and started working at the Philips Research Laboratories in Eindhoven, The Netherlands, in 2000. He has published works in the areas of reconfigurable computing and multiprocessors-on-chip. In 2006, he moved to the Research Department of NXP Semiconductors. He is currently working on the analysis and synthesis of real-

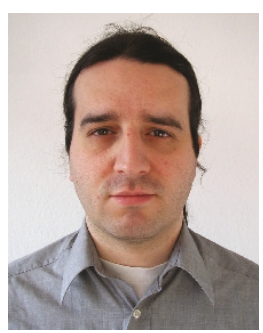
time streaming applications for embedded multiprocessors with network-on-chip communication, and preparing his Ph.D. dissertation at the Eindhoven University of Technology. His other research interests include compilers and programming languages.

Marco J. G. Bekooij received his M.S.E.E. degree from Twente University of Technology in 1995 and his Ph.D. degree from the Eindhoven University of Technology in 2004. He is currently a Senior Researcher at NXP Semiconductors. He has been involved in the design of a channel decoder IC for digital audio broadcasting and a compiler back-end for VLIW processors with distributed register files. His current research

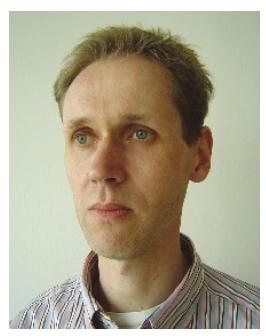
interest is the design and analysis of predictable multiprocessor systems. 